\title{
Review on Fractal Image Compression based on Fast DCT Algorithm
}

\author{
Krishna Chauhan \\ SIRT Bhopal
}

\author{
Anubhuti Khare, Ph.D \\ RGTU Bhopal
}

\begin{abstract}
Fractal image compressions is a lossy compression technique for digital images. It is based on the assumptions that the image redundancies can be efficiently exploited by means of block self-affine transformations .Unlike other compression techniques it considers the interrelations between local (range blocks) and global (domain blocks) data. Use of contractive transform on the space of images encompasses a wide variety of coding scheme. However, the high computational complexity of fractal image encoding greatly restricts its application. This review gives a study of different speed ups using DCT to reduce the searching time
\end{abstract}

\section{Index terms}

Iterated function system, Discrete cosine transform, Self similarity, contractive mapping,

\section{INTRODUCTION}

The idea of fractal image compression was originally introduced by M.F.Barnsley and S.Demko [1]. The algorithm consists of extensive computation[2]. Jacquin proposed the first practical scheme after that [3]. Its base is Partitioned iterated function system (PIFS), which utilizes the self symmetry property. It was block based transformations and has an exhaustive search technique.The procedure of Jacquin-Fisher [4]-[5] algorithm consist of :

1. Use of square blocks size partitioned method to partition the original image into range blocks.

2. To classify given image as per shades, midrange and edge blocks.

3. A search algorithm for domain pool.

Image coding using FIC is done by structure based coding unlike the previous mathods where coding is done through pixel-based methods. Apart from coding FIC has been used in some interesting image problems [6]-[12] and pattern recognition problems such as facial recognition[13]. There were two major issues their algorithm need to improved upon. Firstly to reduce the high computation time and second to use a good classifier so that the overall search space can be reduced. The rest of the paper is organized as follows. In section II the overall process of fractal image coding is discussed. Then in section III DCT based compression with mathematical basics is explained. Different DCT based speedup processes are discussed in IV section.Finally conclusions are made in section V.

\section{FRACTAL IMAGE COMPRESSION}

The Basic idea of fractal image compression is the partitioned iteration function system (PIFS). The main characteristic of iterated function system is -

The redundancy of an image can be used through self transformation on a blockwise basis.IFS approximates the original image by a fractal image.The original image is encoded first and it is then iterated on any initial image to form several images. Finally they are converges to a fractal approximation of the original one.

\section{Image Partition Strategy}

For encoding an $\mathrm{N} \times \mathrm{N}$ gray level image, there are (N/L)2 nonoverlapping blocks forming the range pool $\mathrm{R}$, where $\mathrm{LXL}$ is the coding size of block. Let the contractivity of the fractal coding be a fixed value 2 . Thus, the domain pool is composed of the set of $(\mathrm{N}-2 \mathrm{~L}+1) 2$ overlapping blocks, each of which has size $2 \mathrm{~L} \times 2 \mathrm{~L}$. For the case of $256 \times 256$ image with $8 \times 8$ coding unit, the range pool contains 1024 range blocks, and the domain pool contains 58081 domain blocks of size $16 \times 16$. For each range block in the range pool $\mathrm{R}$, the fractal transformation is constructed by searching in the domain pool for the best match, i.e., the most similar domain block.

The eight Dihedral affine transformations $\mathrm{T}_{\mathrm{K}}, \mathrm{K}=0,1, \ldots, 7$, can be represented by the following matrices:

$\mathrm{T} 0=\left[\begin{array}{ll}1 & 0 \\ 0 & 1\end{array}\right], \quad \mathrm{T} 1=\left[\begin{array}{cc}1 & 0 \\ 0 & -1\end{array}\right]$

$\mathrm{T} 2=\left[\begin{array}{cc}-1 & 0 \\ 0 & 1\end{array}\right], \quad \mathrm{T} 3=\left[\begin{array}{cc}-1 & 0 \\ 0 & -1\end{array}\right]$

$\mathrm{T} 4=\left[\begin{array}{ll}0 & 1 \\ 1 & 0\end{array}\right], \quad \mathrm{T} 5=\left[\begin{array}{cc}0 & 1 \\ -1 & 0\end{array}\right]$

$\mathrm{T} 6=\left[\begin{array}{cc}0 & -1 \\ 1 & 0\end{array}\right], \quad \mathrm{T} 7=\left[\begin{array}{cc}0 & -1 \\ -1 & 0\end{array}\right]$

The eight transform block are denoted by $\mu \mathrm{k}, \mathrm{K}=0,1, \ldots \ldots, 7$, where $\mu 0=\mu$. The transformation $\mathrm{T} 1$ and $\mathrm{T} 2$ flip $\mu$ along the horizontal and vertical lines, respectively, and T3 is the flip along both the horizontal and vertical lines. The transformations T4, T5, T6 and T7 are obtained by flipping T0 , T1, T2 and T3 along the main diagonal line, respectively.

Since the contrast scaling $\mathrm{P}$ and the brightness offset $\mathrm{q}$ are allowed in fractal coding, the fractal affine transformation $\phi$ of $\mathrm{u}(\mathrm{x}, \mathrm{y})$ in D' can be expressed as

$\phi\left[\begin{array}{c}\mathrm{x} \\ \mathrm{y} \\ \mathrm{u}(\mathrm{x}, \mathrm{y})\end{array}\right]=\left[\begin{array}{ccc}\mathrm{a}_{11} & \mathrm{a}_{12} & 0 \\ \mathrm{a}_{21} & \mathrm{a}_{22} & 0 \\ 0 & 0 & \mathrm{P}\end{array}\right]\left[\begin{array}{c}\mathrm{x} \\ \mathrm{y} \\ \mathrm{u}(\mathrm{x}, \mathrm{y})\end{array}\right]+\left[\begin{array}{c}\mathrm{t}_{\mathrm{x}} \\ \mathrm{t}_{\mathrm{y}} \\ \mathrm{q}\end{array}\right](2)$

where $2 \times 2$ sub-matrix $\left[\begin{array}{ll}a_{11} & a_{12} \\ a_{21} & a_{22}\end{array}\right]$ is one of the Dihedral transformation in (1) and (tx, ty) is the location of domain block in the original image. The most similar block is selected as per the MSE( mean square error) between the blocks, defined as follows: 
$\operatorname{MSE}(u, v)=\frac{1}{L^{2}} \sum_{i, j=0}^{L-1}[u(i, j)-v(i, j)]^{2}$

Here two image blocks are denoted by $\mathrm{u}$ and $\mathrm{v}$.

For fractal coding, eight MSE computations between a domain block and a range block are needed due to the eight Dihedral transformations. The best Dihedral transformation corresponds to the minimal MSE obtained by recording the Dihedral index du such that

$$
\mathrm{du}=\operatorname{argK} \mathrm{K}_{\min }\left\{\operatorname{MSE}\left(\mathrm{P}_{\mathrm{k}} \mathrm{u}_{\mathrm{k}}+\mathrm{q}_{\mathrm{k}}, \mathrm{v}\right): \mathrm{k}=0,1, \ldots . .7\right\}
$$

where $\mathrm{P}_{\mathrm{k}}$ and $\mathrm{u}_{\mathrm{k}}$ are contrast scaling and brightness offset, respectively.

For each $\mathrm{k}$, optimal $\mathrm{p}_{\mathrm{k}}{ }^{*}$ and $\mathrm{q}_{\mathrm{k}}{ }^{*}$ can be computed directly as

$$
\begin{array}{r}
\mathrm{p}_{\mathrm{k}}{ }^{*}=\frac{\mathrm{L}^{2}\left(\mathrm{u}_{\mathrm{k}}{ }^{*}, \mathrm{v}\right)-\left(\mathrm{u}_{\mathrm{k}}{ }^{*}, \mathrm{e}\right) \cdot(\mathrm{v}, \mathrm{e})}{\mathrm{L}^{2}\left(\mathrm{u}_{\mathrm{k}}{ }^{*}, \mathrm{v}\right)-\left(\mathrm{u}_{\mathrm{k}}{ }^{*}, \mathrm{e}\right)^{2}}, \\
\mathrm{q}_{\mathrm{k}}{ }^{*}=\frac{1}{\mathrm{~L}^{2}}\left[(\mathrm{v}, \mathrm{e})-\mathrm{P}_{\mathrm{k}}\left(\mathrm{u}_{\mathrm{k}}, \mathrm{e}\right)\right]
\end{array}
$$

Where (...) is the Euclidean inner product and $\mathrm{e}=$ $\left[\begin{array}{llll}1 & 1 & \ldots & .\end{array}\right]^{\mathrm{T}}$.

Finally, as $\mathrm{v}$ runs over all of the range blocks in $\mathrm{R}$, the encoding process is completed. Jacquin also suggested here a two level partition [3].One a large cell size ( parent cell) and another a small cell size ( child cell).Here the parent cell can be split up into four non overlapping child cells. This decision is made during encoding only.So basically it leads to advantages of small block size, which can be easily analyze and encode also , larger block size where redundancies can be easily exploited, to higher compression ratios.

To decode, we first make up the 1024 affine transformations from the compression codes and choose any image as the initial one. Then, perform the 1024 affine transforms on the image to obtain a new image, and proceeds recursively. According to PIFS Theorem and the Collage theorem, the sequence of images will converge. The stopping criterion of the recursion is designed according to user's application. The final image is the retrieved image of fractal coding.

\section{DISCRETE COSINE TRANSFORM of IFS}

Full search for domain block is very time consuming process.DCT based FIC is introduced [14].In this fast encoding algorithm whenever a new entry for domain block is done, the error calculations between range block and dihedral symmetries of domain block are obtained simultaneously in frequency domain. So redundancies are removed by ignoring higher indices components in further processing.

All matrix data calculations for performing discrete cosine transform is same as given above. Here fixed contractivity of $1 / 2$ is used in general. For each domain block there are eight orientations which are obtained by the use of the identity map, reflections about the center vertical line, reflections about the center horizontal line and the center point of the original block, plus the additional flip about the line $\mathrm{y}=\mathrm{x}$ for each of the four cases above. There are Dihedral-group transformations. Given a gray level image $f(x, y)$ the transformation for the fractal image compression is of the form given in (2)

One arranges the $2 \times 2$ sub matrix for these eight orientations as follows:

Case 1: T0 $=\left[\begin{array}{ll}\frac{1}{2} & 0 \\ 0 & \frac{1}{2}\end{array}\right]$,

Case 5: T0 $=\left[\begin{array}{ll}0 & \frac{1}{2} \\ \frac{1}{2} & 0\end{array}\right]$

Case 2: T0 $=\left[\begin{array}{cc}-\frac{1}{2} & 0 \\ 0 & \frac{1}{2}\end{array}\right]$

Case 6: T0 $=\left[\begin{array}{cc}0 & -\frac{1}{2} \\ \frac{1}{2} & 0\end{array}\right]$

Case 3: T0 $=\left[\begin{array}{cc}\frac{1}{2} & 0 \\ 0 & -\frac{1}{2}\end{array}\right]$

Case 7: T0 $=\left[\begin{array}{cc}0 & \frac{1}{2} \\ -\frac{1}{2} & 0\end{array}\right]$

Case 4: T0 $=\left[\begin{array}{cc}-\frac{1}{2} & 0 \\ 0 & -\frac{1}{2}\end{array}\right]$ Case 8: $\mathrm{T} 0=\left[\begin{array}{cc}0 & -\frac{1}{2} \\ -\frac{1}{2} & 0\end{array}\right]$

Here the contractivity is taken as half.Note the case 1, 6, 4 and 7 are the same as those obtained by counterclockwise rotations about the original block with angle $0^{\circ}, 90^{\circ}, 180^{\circ}$ and $270^{\circ}$ respectively. Formulas of the DCT of each orientation are formed in terms of the DCT of the original block. Using these, formulas, one is able to group the data under consideration and eliminate repeated calculation of the different operations and, thus reduce the computation time. It is shown[15], that the DCT of an $\mathrm{L} \times \mathrm{L}$ image block $f(i, j), i, j=0,1, \ldots . \mathrm{L}-1$ is given by

$\mathrm{F}(\mathrm{m}, \mathrm{n})=$

$\frac{2}{\mathrm{~L}} \mathrm{C}_{\mathrm{m}} \mathrm{C}_{\mathrm{n}} \sum_{\mathrm{i}=0}^{\mathrm{L}-1} \sum_{\mathrm{i}=0}^{\mathrm{L}-1} \mathrm{f}(\mathrm{i}, \mathrm{j}) \cos \left(\frac{(2 \mathrm{i}+1) \mathrm{m} \pi}{2 \mathrm{~L}}\right) \cdot \cos$

$\left(\frac{(2 \mathrm{i}+1) \mathrm{n} \pi}{2 \mathrm{~L}}\right)$

Where $m, n=0,1, \ldots \ldots L-1$ and

$\mathrm{Ck}= \begin{cases}\frac{1}{\sqrt{2}}, & \mathrm{k}=0 \\ 1, & \mathrm{k} \neq 0 .\end{cases}$

Initially DCT for $\mathrm{x}$ and $\mathrm{y}$ is evaluated for the line $\mathrm{x}=(\mathrm{L}-1) / 2$ and the line $y=(L-1) / 2$. The change of the coordinates is about the line $\mathrm{x}=(\mathrm{L}-1) / 2$. The new coordinates $(\mathrm{u}, \mathrm{v})$ is given by

$\left[\begin{array}{c}\mathrm{u}-\frac{\mathrm{L}-1}{2} \\ \mathrm{v}\end{array}\right]=\left[\begin{array}{cc}-1 & 0 \\ 0 & 1\end{array}\right]\left[\begin{array}{c}\mathrm{u}-\frac{\mathrm{L}-1}{2} \\ \mathrm{y}\end{array}\right]$

And solved as

$\mathrm{u}=-\mathrm{x}+\mathrm{L}-1$ and $\mathrm{v}=\mathrm{y}$.

Let $\mathrm{g} 1(\mathrm{u}, \mathrm{v})$ be the new image block that is obtained from $\mathrm{f}(\mathrm{m}$, n) by this reflection. One writes

$g 1(u, v)=g 1(u(x, y), v(x, y))=f(x, y)$.

By (7) and (9), the DCT of $\mathrm{g} 1$ is thus computed as 


$$
\begin{aligned}
& \mathrm{G} 1(\mathrm{~m}, \quad \mathrm{n} \\
& \mathrm{C}_{\mathrm{m}} \mathrm{C}_{\mathrm{n}} \sum_{\mathrm{u}=0}^{\mathrm{L}-1} \sum_{\mathrm{v}=0}^{\mathrm{L}-1} \mathrm{~g} 1(\mathrm{u}, \mathrm{v}) \cos \left(\frac{(2 \mathrm{i}+1) \mathrm{m} \pi}{2 \mathrm{~L}}\right) \cdot \cos \\
& \left(\frac{(2 \mathrm{i}+1) \mathrm{n} \pi}{2 \mathrm{~L}}\right) \\
& = \\
& \mathrm{C}_{\mathrm{m}} \mathrm{C}_{\mathrm{n}} \sum_{-\mathrm{x}+\mathrm{L}-1=0}^{\mathrm{L}-1} \sum_{\mathrm{y}=0}^{\mathrm{L}-1} \mathrm{f}(\mathrm{x}, \mathrm{y}) \cos \left(\frac{[2(-\mathrm{x}+\mathrm{L}-1)+1] \mathrm{m} \pi}{2 \mathrm{~L}}\right) \\
& \cos \left(\frac{(2 \mathrm{y}+1) \mathrm{n} \pi}{2 \mathrm{~L}}\right) \\
& =\mathrm{C}_{\mathrm{m}} \mathrm{C}_{\mathrm{n}} \sum_{\mathrm{x}=0}^{\mathrm{L}-1} \sum_{\mathrm{y}=0}^{\mathrm{L}-1} \mathrm{f}(\mathrm{x}, \mathrm{y}) \cos \left(\frac{(2 \mathrm{x}+1) \mathrm{m} \pi}{2 \mathrm{~L}}+\mathrm{m} \pi\right) \cdot \mathrm{c} \\
& \operatorname{os}\left(\frac{(2 \mathrm{y}+1) \mathrm{n} \pi}{2 \mathrm{~L}}\right)
\end{aligned}
$$$$
=(-1)^{\mathrm{m}} \mathrm{C}_{\mathrm{m}} \mathrm{C}_{\mathrm{n}} \sum_{\mathrm{x}=0}^{\mathrm{L}-1} \sum_{\mathrm{y}=0}^{\mathrm{L}-1} \mathrm{f}(\mathrm{x}, \mathrm{y}) \cos \left(\frac{(2 \mathrm{x}+1) \mathrm{m} \pi}{2 \mathrm{~L}}\right) .
$$$$
\cos \left(\frac{(2 \mathrm{y}+1) \mathrm{n} \pi}{2 \mathrm{~L}}\right)
$$$$
=(-1)^{\mathrm{m}} \mathrm{F}(\mathrm{m}, \mathrm{n}) \text {. }
$$$$
\text { (10) }
$$

For the case of the reflection about $y=(\mathrm{L}-1) / 2$, let $\mathrm{g} 2(\mathrm{u}, \mathrm{v}) \mathrm{be}$ the new image block obtained from $\mathrm{f}(\mathrm{m}, \mathrm{n})$, by this reflection and $\mathrm{G} 2$ be the DCT of g2. Similar computation in (10) lead to

$$
\mathrm{G} 2(\mathrm{~m}, \mathrm{n})=(-1)^{\mathrm{m}} \mathrm{F}(\mathrm{m}, \mathrm{n}) \text {. }
$$

Next one compute the DCT for the case of the flip about the line $y=x$. Consider the change of coordinates by flipping the original block about the line $y=x$. the new coordinate $(u, v)$ is thus given by

$$
\left[\begin{array}{l}
u \\
v
\end{array}\right]=\left[\begin{array}{ll}
1 & 0 \\
0 & 1
\end{array}\right]\left[\begin{array}{l}
\mathrm{x} \\
\mathrm{y}
\end{array}\right]
$$

And solved as

$$
\mathrm{u}=\mathrm{y} \text { and } \mathrm{v}=\mathrm{x}
$$

Let $h(u, v)$ be the new image block obtaioned from $f(m, n)$ by this flip. One writes

$$
h(u, v)=h(u(x, y), v(x, y))=f(x, y)
$$

By (3) and (9) thus DCT of $h$ is computed as

$\mathrm{H}(\mathrm{m}$

n)

$$
\begin{aligned}
& \mathrm{C}_{\mathrm{m}} \mathrm{C}_{\mathrm{n}} \sum_{\mathrm{u}=0}^{\mathrm{L}-1} \sum_{\mathrm{v}=0}^{\mathrm{L}-1} \mathrm{~h}(\mathrm{u}, \mathrm{v}) \cos \left(\frac{(2 \mathrm{u}+1) \mathrm{m} \pi}{2 \mathrm{~L}}\right) \cdot \cos \\
& \left(\frac{(2 \mathrm{v}+1) \mathrm{n} \pi}{2 \mathrm{~L}}\right) \\
& = \\
& \mathrm{C}_{\mathrm{m}} \mathrm{C}_{\mathrm{n}} \sum_{\mathrm{y}=0}^{\mathrm{L}-1} \sum_{\mathrm{x}=0}^{\mathrm{L}-1} \mathrm{f}(\mathrm{x}, \mathrm{y}) \cos \left(\frac{(2 \mathrm{y}+1) \mathrm{m} \pi}{2 \mathrm{~L}}\right) \cdot \cos \\
& \left(\frac{(2 \mathrm{x}+1) \mathrm{n} \pi}{2 \mathrm{~L}}\right) \\
& =\mathrm{C}_{\mathrm{m}} \mathrm{C}_{\mathrm{n}} \sum_{\mathrm{x}=0}^{\mathrm{L}-1} \sum_{\mathrm{y}=0}^{\mathrm{L}-1} \mathrm{f}(\mathrm{x}, \mathrm{y}) \cos \left(\frac{(2 \mathrm{x}+1) \mathrm{n} \pi}{2 \mathrm{~L}}\right) . \\
& \cos \left(\frac{(2 \mathrm{y}+1) \mathrm{m} \pi}{2 \mathrm{~L}}\right) \\
& =\mathrm{F}(\mathrm{n}, \mathrm{m}) .
\end{aligned}
$$

$=\quad$ Now, let $\mathrm{fk}, \mathrm{k}=1,2, \ldots, 8$ denote the image block for case $\mathrm{k}$ and FkThus by the formulas (10), (11) and (14), it is easily shown that

$$
\begin{aligned}
& \mathrm{F} 2(\mathrm{~m}, \mathrm{n})=(-1)^{\mathrm{m}} \mathrm{F}_{1}(\mathrm{~m}, \mathrm{n}) . \\
& \mathrm{F} 3(\mathrm{~m}, \mathrm{n})=(-1)^{\mathrm{m}} \mathrm{F}_{1}(\mathrm{~m}, \mathrm{n}) . \\
& \mathrm{F} 4(\mathrm{~m}, \mathrm{n})=(-1)^{\mathrm{m}+\mathrm{n}} \mathrm{F}_{1}(\mathrm{~m}, \mathrm{n}) .
\end{aligned}
$$

And

$$
\begin{aligned}
& \mathrm{F} 5(\mathrm{~m}, \mathrm{n})=(-1)^{\mathrm{m}} \mathrm{F}_{1}(\mathrm{~m}, \mathrm{n}) \\
& \mathrm{F} 6(\mathrm{~m}, \mathrm{n})=\mathrm{F}_{2}(\mathrm{n}, \mathrm{m})=(-1)^{\mathrm{n}} \mathrm{F}_{1}(\mathrm{n}, \mathrm{m}) \\
& \mathrm{F} 7(\mathrm{~m}, \mathrm{n})=\mathrm{F}_{3}(\mathrm{n}, \mathrm{m})=(-1)^{\mathrm{m}} \mathrm{F}_{1}(\mathrm{n}, \mathrm{m}) \\
& \mathrm{F} 8(\mathrm{~m}, \mathrm{n})=\mathrm{F}_{4}(\mathrm{n}, \mathrm{m})=(-1)^{\mathrm{m}+\mathrm{n}} \mathrm{F}_{1}(\mathrm{n}, \mathrm{m})
\end{aligned}
$$

The optimum values of $\mathrm{p}$ and $\mathrm{q}$ are founded by least square method in baseline algorithm of Jacquin and Fisher.The more DCT coefficients are used more accurate will be the values of $p$ and q.In baseline algorithm for encoding 8 bits are used for location or translations for $\mathrm{x}$ and $\mathrm{y}$ plane, 5 bits for contrast, 7 bits for brightness and 3 bits for dihedral transform. In this way total 31 bits are used for a coding unit or range block. Truong concluded by using this fast algorithm the speed of algorithm can be increased 6 times faster [16]. Here all unwanted calculations are removed for dihedral symmetries.Further more the complexity of DCT inner product is reduced.

\section{SPEED UPS THROUGH DCT}

- Through good classification scheme: Certain classifications methods can be used to further improve the time constraints of algorithm, for reducing the size of domain block. During the encoding process, the range blocks and domain blocks are classified first. Then, each range block is limited to search in the corresponding domain class to find the best match. Since the searching space is reduced, the encoding speed is improved. Here the quality of retrieved images is almost same as that of full search method.

- Hybrid technique using DWT, DCT and Huffman coding : A hybrid DCT with DWT can also compress images to a better extent[17]. Here Discrete wavelet transform is used at first level. They are decomposed into low and high frequency components, out of which only low frequency components are used while high frequency components are discarded. This process can be further repeated for next level depends upon the tolerance of retrieved image quality. Then only low frequency components are decomposed using DCT.As most of the energy is concentrated over lower indices in DCT. The analysis of the previous conducted tests has stimulated that the energy packing attribute of DCT coefficients is very useful in classifying the domain and range blocks and isometric process prediction.Using the color model YUV leads to good compression ratio when it is compared with the case of using RGB.

- $\quad$ DCT parallel encoding and recombination: Despite of using a fractal encoder alone a parallel DCT and fractal encoder can be used to get a better decompression. Here these two encoders are used to preserve different details of image.DCT encoder for the detail information of the image while fractal for getting a good quality of edge and smoothness variation. It has been shown that this hybrid 
technique gives better results as using one decoder at a time. Here a uniform algorithm is suitable for real time VLSI implementation.

- Using no full search algorithm : In 2007 Salarian and Hassanpour proposed another DCT based fractal coding[18]. Here for each range block one domain block is considered, and searched only for contrast scaling. Using this technique we can further reduce the size of coding unit as limited number of comparisons are there. Therefore the quality of decoded image can be improved while the compression rate is maintained. Further Chong and Zhi-liang Zu suggested [19] despite of full search for each domain block it is enough to classify these blocks into certain classes and then only search for same domain and range blocks. This scheme greatly reduced the searching time as well as PSNR.

\section{CONCLUSION}

This paper has surveyed significant advancement in different steps of FIC using DCT.Further work is needed to enhance the DCT filter method by using hierarchal partition schemes (Quadtree or Horizontal-Vertical partition) to attain higher compression ratios. Irregular partition method outperforms in comparision to these two method.The major improvement needed is to reduce the coding time. Non uniform quantized classification descriptor can be used to address the domain and range blocks. The arrangements of domain and range blocks can further remove the problems of coding complexity. The most significant advantage of DCT based compression techniques is, there are no further degradation in image quality for same compression ratios.

\section{REFERENCES}

[1] M. F. Barnsley and S. Demko, "Iterated function systems and the global construction of fractals," Proc. R. Soc. Lond. A, vol. 399, pp. 243-275, 1985.

[2] M. F. Barnsley and L. P. Hurd, Fractal Image Compression. Wellesley, MA: A. K. Peter, 1993.

[3] A. E. Jacquin, "Image coding based on a fractal theory of iterated contractive image transformations," IEEE Trans. Image Processing, vol. 1, pp. 18-30, Jan. 1992.

[4]M. F. Barnsley, "Methods and apparatus for image compression by iterated function systems," U.S. Patent 4 941 193, July 1990.

[5] S. Graf, "Barnsley's scheme for the fractal encoding of images," J. Complex., vol. 8, pp. 72-78, 1992.

[6] M. Pi, M. K. Mandal, and A. Basu, "Image retrieval based on histogram of fractal parameters,"IEEE Trans. Multimedia, vol. 7, no. 4,pp. 597-605, Aug. 2005.
[7] J. H. Jeng, C. C. Tseng, and J. G. Hsieh, "Study on Huber fractal image compression," IEEE Trans. Image Process., vol. 18, no. 5, pp. 995-1003, May 2009.

[8] M. Ghazel, G. H. Freeman, and E. R. Vrscay, "Fractal image denoising," IEEE Trans. Image Process., vol. 12, no. 12 , pp. $1560-1578$, Dec. 2003

[9] M. Ghazel, G. H. Freeman, and E. R. Vrscay, "Fractalwavelet image denoising revisited," IEEE Trans. Image Process., vol. 15, no. 9, pp. 2669-2675, Sep. 2006.

[10] S. S. Wang and S. L. Tsai, "Automatic image authentication and recovery using fractal code embedding and image inpainting," Pattern Recognit. vol. 41, no. 2, pp. 701-712, 2008.

[11] S. G. Lian, "Image authentication based on fractal features," Fractals vol. 16, no. 4, pp. 287-297, 2008.

[12] K. T. Lin and S. L. Yeh, "Encrypting image by assembling the fractal- image addition method and the binary encoding method," Opt. Commun.vol. 285, no. 9, pp. 2335-2342, 2012

[13]X. Tang and C. Qu, "Facial image recognition based on fractal image encoding, Bell Labs Tech. J. , vol. 15, no. 1, pp. 209-214, 2010

[14] Arnaud E. Jacquin "Image Coding Based On "A Fractal Theory Of Iterated Contractive Image Transformations" IEEE transactions on image processing. vol.1 no. 1. january 1992 .

[15]N. I. Cho and S. U. Lee, "Fast algorithm and implementation of 2-D discrete cosine transform," IEEE Trans. Circuits Syst., vol. 38, Mar. 1991

[16] Trieu-Kien Truong, Jyh-Horng Jeng, Irving S. Reed, P. C. Lee, and Alan Q. Li"A Fast Encoding Algorithm for Fractal Compression Using the DCT Inner Product Image" IEEE TRANSACTIONS ON IMAGE PROCESSING, VOL. 9, NO. 4, APRIL 2000

[17] Harjeetpal singh, Sakhi Sharma "Hybrid Image Compression Using DWT, DCT \& Huffman Encoding Techniques" International Journal of Emerging Technology and Advanced Engineering (ISSN 22502459, Volume 2, Issue 10, October 2012)

[18]Salarian, M. and ; Hassanpour, H.," A new fast no search fractal image compression in DCT domain" Machine Vision, 2007. ICMV 2007. International Conference 62-66 (2007).

[19] Chong Fu ; Sch. of Inf. Sci. \& Eng., Northeastern Univ., Shenyang, China ; Zhi-liang Zhu "A DCT-Based Fractal Image Compression Method" Chaos-Fractals Theories and Applications, 2009. IWCFTA '09. International Workshop 439-443 (2009) 